\title{
Discrete-Time Pricing and Optimal Exercise of American Perpetual Warrants in the Geometric Random Walk Model
}

\author{
Robert J. Vanderbei • Mustafa Ç. Pınar • \\ Efe B. Bozkaya
}

Published online: 26 September 2012

(C) Springer Science+Business Media New York 2012

\begin{abstract}
An American option (or, warrant) is the right, but not the obligation, to purchase or sell an underlying equity at any time up to a predetermined expiration date for a predetermined amount. A perpetual American option differs from a plain American option in that it does not expire. In this study, we solve the optimal stopping problem of a perpetual American option (both call and put) in discrete time using linear programming duality. Under the assumption that the underlying stock price follows a discrete time and discrete state Markov process, namely a geometric random walk, we formulate the pricing problem as an infinite dimensional linear programming (LP) problem using the excessive-majorant property of the value function. This formulation allows us to solve complementary slackness conditions in closedform, revealing an optimal stopping strategy which highlights the set of stock-prices where the option should be exercised. The analysis for the call option reveals that such a critical value exists only in some cases, depending on a combination of statetransition probabilities and the economic discount factor (i.e., the prevailing interest rate) whereas it ceases to be an issue for the put.
\end{abstract}

Keywords American perpetual warrants · Pricing · Optimal stopping · Optimal exercise $\cdot$ Random walk $\cdot$ Linear programming $\cdot$ Duality

M.Ç. Pınar was supported in part by TUBITAK Grant 107K250.

\footnotetext{
R.J. Vanderbei

Department of Operations Research and Financial Engineering, Princeton University, Princeton, USA

e-mail: rvdb@princeton.edu

M.Ç. Pınar ( $\varangle)$

Department of Industrial Engineering, Bilkent University, Ankara, Turkey

e-mail: mustafap@bilkent.edu.tr

E.B. Bozkaya

Faculty of Administrative Sciences, Sabancı University, Istambul, Turkey
} 


\section{Introduction}

The subject of determining correct market prices for options (a.k.a warrants) is a well-known and widely studied branch of mathematical finance. Valuation of options has consistently been in the center of the derivative pricing literature. One of the most significant distinctions within the literature in the field is the study of European versus American type options. In this paper we study the valuation of American options without an expiration date written on stocks that follow a geometric random walk.

Comprehensive treatments of option pricing can be found in Hobson [7] and specifically for American options in Myneni's [9] survey.

The problem of determining correct prices for American type contingent claims was first handled by McKean in a continuous-time Geometric Brownian motion setting upon a question posed by Samuelson (see Appendix of [10]). In his response, McKean transformed the problem of pricing American options into a free boundary problem and gave a closed form solution for perpetual American options. The formal treatment of the problem from an optimal stopping perspective was later done by Moerbeke [15] and Karatzas [8], who used hedging arguments for financial justification. Wong, in a recent study, has collected the optimal stopping problems arising in the financial markets [18]. A textbook treatment of American options in continuous time can be found in Chap. 8 of [11].

In this paper, following on our previous work [16], which is more of a pedagogical nature in the more straightforward case of a simple random walk model, we provide an alternative approach to solving the pricing problem of perpetual American call and put options when the underlying stock follows a geometric random walk, a special case of discrete-time/discrete-state Markov processes. Our objective is to determine the optimal stopping region(s) for exercising the option contract. It is well known that the value function of an optimal stopping problem for a Markov process is the minimal excessive function majorizing the pay-off of the reward process (see $[1,5]$ ). The value function, then, can be obtained by solving an infinite dimensional linear program using the duality theory of linear programming. This approach is taken, for instance, in [14] to treat singular stochastic control problems. The solution approach for the call option relies on finding the increasing solution to a certain difference equation and then identifying the value function in a specific form. For the put option we use the decreasing solution to this difference equation. This approach mirrors the work in continuous-time/continuous-state processes as reported in $[6,12,13,17]$. In [6] in particular an LP embedding and duality is used in the analysis of optimal stopping problems for one-dimensional diffusions. In discrete time, a semi-explicit solution for the call problem for general random walks is given in [2] while a more recent solution for more general reward functions is derived in [3] for processes with stationary independent increments. In an unpublished recent Master's thesis [4] the problem of optimal stopping is treated in a setting identical to ours, but using different methods, namely the method of measure transformation. We note that this reference takes into account the ambiguity on probability measure by allowing multiple priors. In the case of a single prior, one solves problems identical to ours.

In a recent paper, Vanderbei and Pinar [16] use the approach of linear programming to propose an alternative method for the pricing of American perpetual call 
warrants. Under mild assumptions, they find that the optimal stopping region can be characterized by a critical threshold which, when reached, leads to the decision to exercise. In this paper, we extend the analysis on simple random walks by providing a more general optimal stopping criterion and giving a solution to the geometric random walk case for both the call and the put options. Although the geometric random walk case was suggested as an exercise in [16], the analysis turns out to be quite involved and sufficiently different from the simple random walk case to warrant a separate circulation of the results of the present paper.

A call option is a contract giving the right, but not the obligation, to buy an asset at a certain time in the future for a previously agreed price, whereas, a put option is the right, but not the obligation, to sell an asset in a similar fashion. The agreed price is called the strike price. The issuer of an option contract is said to write the option and the date at which the right of exercising an option expires is known as the maturity date. When the holder of an option uses the contract, which means (s)he buys or sells the asset at the strike price, we say that the holder has exercised the option. In a given state of the world, the amount that the holder of the option gains or loses is captured as a function of the underlying's pay-off. This is defined as the option pay-off. In the remainder of this work, we will use $S$ for the strike price, $T$ for the maturity date and the real-valued function $f: E \rightarrow \mathbb{R}$ for the option pay-off where $E$ is the set of all possible states of the world.

An option whose exercise is only possible at the maturity date $T$ is said to be a European type option while for the American type options, early exercise is allowed in the period $[0, T]$. These two types of options are known as plain vanilla options and they form the basis of option pricing literature. The holder of the American option observes the price of the underlying throughout the life of the option and decides on an exercise time that maximizes his earnings.

Assume that for some state of the world $x \in E$, the market value of the underlying is captured by the variable $h(x)$. For the owner of a call option, if $h(x)$ is greater than the strike price $S$ at the maturity date, it is meaningful for the holder to exercise the option for an immediate gain of $h(x)-S$, since the contract gives her the right to buy a unit of the underlying at the price $S$. Then, by selling this unit for its market value $h(x)$, the owner can realize the specified gain. If, the price of the underlying, however, is lower than $S$, it will not be profitable to exercise the option because the same asset is already available cheaper in the exchange market. For a call option, the pay-off function corresponds to:

$$
f(x)=\max \{h(x)-S, 0\}=(h(x)-S)_{+} .
$$

The pay-off of the American call option treated in this paper is modeled using the real valued function $f: E \times T \rightarrow \mathbb{R}$, where $T$ is an index set representing time. We reserve the symbol $X_{t}(x)$ for the value of the underlying asset at time $t$ and state $x$, and define $f_{t}(x)$ to be the image of $(x, t) \in E \times T$ for some $x \in E$ and $t \in T$.

The holder of an American type option is interested in determining the correct moment to exercise the contract. The characterization of optimal exercising rules where the decision is made with respect to the expected pay-off in the future is the main objective of this work. Our problem, therefore, is to obtain such states of the world where it is no longer meaningful for the trader to retain the rights to the underlying. 
In order to make a decision, the trader must possess the knowledge of the best possible pay-off in the future, at each state of the world. Having such information allows the trader to compare what he can get at a particular point in time to the best he can do in the future. Delaying the decision to exercise when the best future pay-off cannot beat the immediate pay-off will clearly be suboptimal, due to the time value of money.

We call the best future pay-off at each state of the world the value of the option. Suppose the underlying stock follows a stochastic process $X_{t}$ on the state space $E$. For any initial state $x \in E$ and at any future time $t>0$, we can denote the expected pay-off with $\mathbb{E}\left[f\left(X_{t}\right) \mid X_{0}=x\right]$. The maximum of such functions over all stopping times $\tau$ (recall that a stopping time with respect to a sequence of random variables $X_{1}, X_{2}, \ldots$ is a random variable $\tau$ with the property that for each $t$, the occurrence or non-occurrence of the event $\tau=t$ depends only on the values of $X_{1}, X_{2}, \ldots, X_{t}$ ) will be our value function, which will be denoted with $v$. In mathematical terms, a definition for $v$ is

$$
v(x)=\max _{\tau \in \mathcal{T}} \mathbb{E}_{x}\left[\alpha^{\tau} f\left(X_{\tau}\right)\right],
$$

where $\mathcal{T}$ represents the set of all stopping times. Our problem is to find a subset $O P T$ (termed the support set in [1,5]) of the state space $E$ where for all $x \in O P T$ we have $v(x)=f(x)$. Note that it is not possible to have $v(x)<f(x)$ since the time-index set includes time zero. Thus, for any $x \notin O P T$ we must have $v(x)>f(x)$ which means that the best expected future pay-off is larger than what is readily available. Then, the correct decision must be to wait further to exercise the contract. It may also be the case (as in the case of a perpetual call option) that it is optimal to never exercise the option, i.e. $\tau=\infty$ in which case the pay-off is zero.

In this work, we determine the correct value function and the set $O P T$ to understand when to make an optimal stopping decision. In the rest of the paper, we make the following assumptions:

1. The stock price process follows a discrete time and discrete state random walk.

2. There is a fixed discount rate $\alpha \in(0,1)$ per period due to the time value of money.

3. The option contract under study may be written without an expiration date.

The rest of this paper is organized as follows. In Sect. 2, we review the mathematical background relevant to our analysis. In Sect. 3, we present the geometric random walk model for asset prices, and carry out the analysis for the call option in Sect. 4. In Sect. 5, we give numerical illustrations. In Sect. 6, we extend the analysis to put options of American perpetual type giving the essential points without repeating all the details.

\section{Background}

In this section we present mathematical definitions and tools to lay the groundwork for deriving rules for exercising perpetual American type options. The majority of results in this section are from the optimal stopping literature on stochastic processes, 
especially on the ramifications of the Markov hypothesis. The reader is encouraged to see [5] for a thorough yet readable treatment of Markov processes. The notation throughout the section is borrowed from Çınlar's introductory text on stochastic processes [1].

Let the triplet $(\Omega, \mathcal{F}, \mathbb{P})$ be a probability space. We start with the classic definitions of stochastic processes and Markov processes.

Definition 2.1 A stochastic process $X=\left\{X_{t}, t \in T\right\}$ with the state space $E$ is a collection of $E$-valued random variables indexed by a set $T$, often interpreted as the time. We say that $\boldsymbol{X}$ is a discrete-time stochastic process if $T$ is countable and a continuoustime stochastic process if $T$ is uncountable. Likewise, $X$ is called a discrete-state stochastic process if $E$ is countable and called a continuous-state stochastic process if $E$ is an uncountable set.

For any $i \in E$, we define $\mathbb{P}_{i}\left(X_{t}\right)$ to be the conditional probability distribution of the stochastic process at time $t$ conditional on the initial state $i$. Similarly $\mathbb{E}_{i}\left(X_{t}\right)$ is defined to be the conditional expectation of the value of the stochastic process at time $t$ conditional on the initial state $i$. In this work we assume that the stock prices follow a stochastic process with the Markov property. We use discrete state Markov processes defined on $\mathbb{R}^{+}$to model stock prices.

The study of excessive functions is of significant importance in the optimal stopping literature. These functions are tools to connect the underlying stochastic process to the outcomes associated with the movement of the process in time.

Definition 2.2 A real-valued function $g: E \rightarrow \mathbb{R}$ is called the reward function of a stochastic process $\boldsymbol{X}$.

A reward function defined on the states of the process represents a quantity acquired once the process enters a particular state in time. Our motivation in considering reward functions comes from the need to model the pay-off of an option contract depending on the pay-off of the underlying stock.

Let $\alpha \in[0,1]$ be a constant that denotes the discount factor. Next, we introduce the family of $\alpha$-excessive functions which plays a key role in characterizing the value function of a stochastic process.

Definition 2.3 Let $f$ be a finite-valued function defined on a countable state space $E$ and $P$ be a transition matrix. The function $f$ is said to be $\alpha$-excessive provided that $f \geq 0$ and $f \geq \alpha P f$. If $f$ is 1 -excessive, it is simply called excessive.

Note that a reward function on $E$ need not necessarily be $\alpha$-excessive though, as we will later see, the value function of a stochastic process must be $\alpha$-excessive. To define this value function, we will look at the optimal stopping problem of a Markov process.

Suppose we have the Markov process $X$, the transition matrix $P$ and the reward function $f$ defined on the state space $E$. Let $t=0$ denote time zero, the initial period 
at the beginning of analysis and suppose $X_{0}=i$. A valid measure of assessing a particular state's value can be defined as:

$$
\sup _{\tau} \mathbb{E}_{i}\left[\alpha^{\tau} f\left(X_{\tau}\right)\right]
$$

which gives the supremum of the discounted expected future rewards over all stopping times $\tau$ when the initial state is $i$. In other words, it gives the highest expected value of future rewards when the current state of the process is $i$. Thus, we can define the value of the optimal stopping problem as a function from $E$ to the real numbers, which gives the highest possible expected pay-off per state.

Definition 2.4 The real valued function $v$ on $E$ given by

$$
v(i)=\sup _{\tau} \mathbb{E}_{i}\left[\alpha^{\tau} f\left(X_{\tau}\right)\right]
$$

is called the value function of the optimal stopping problem associated with the Markov process $X$ and the reward function $f$.

In order to make a stopping decision, we need to determine the set of states, say $O P T \subset E$, such that $v(j)=f(j), \forall j \in O P T$. Note that for any state with this property, it is unwise, in terms of the expected future reward, to hold off from stopping in the hopes of higher future gains. Since the participant will never get a better value in the future, on average, the correct decision is to stop immediately and collect the reward. In our setting, this corresponds to exercising the option. An optimal strategy can, therefore, be characterized as to exercise the option as soon as the underlying stock process attains a value in $O P T$. Note, however, that in the case of a call option, the option may never be exercised, i.e., $\tau=\infty$. In infinite state spaces, it may also occur that the first hitting time of the support set does not give an optimal stopping time even if the value function is finite. Numerical examples illustrating such situations can be found e.g. on p. 108 of [5].

With these definitions from the optimal stopping literature in mind, we note that the problem of pricing a perpetual American option and determining the optimal stopping strategy is equivalent to computing a value function for the underlying stockprice process and determining the set of states where the value function is equal to the pay-off of the option. The essence of our study will be the application of the following key result allowing the problem to be formulated as a linear program.

Theorem 2.1 The value function $v$ is the minimal $\alpha$-excessive function greater than or equal to the pay-off function $f$.

Proof See [5], p. 105 or [1], p. 221. 
Now, suppose that both $E$ and $T$ are countable. We can use the above result to convert the problem to the following LP using the definition of an excessive function:

$$
\begin{array}{ll}
\min & \sum_{i \in E} v(i) \\
\text { s.t. } & v(i) \geq f(i), \quad i \in E \\
& v(i) \geq \alpha P v, \quad i \in E \\
& v(i) \geq 0, \quad i \in E .
\end{array}
$$

\section{A Geometric Random Walk Model on $\mathbb{R}$ for the Stock Price Process}

Let $\varphi$ be a real number slightly greater than 1 and $X_{0}>0$ be the current price of the underlying stock. Without loss of generality, we take $t=0$ as the current time. Consider the state space $E_{2}=\left\{X_{0} \cdot \varphi^{j}: j \in \mathbb{Z}\right\}$. Note that $\lim _{j \rightarrow-\infty} \varphi^{j}=0$, thus, our state space $E_{2}$ consists of positive-valued elements constructed as a geometric series. We define the stock-price process as the collection of $E_{2}$-valued random variables $\left\{X_{t}: t \in \mathbb{N}\right\}$. Let $t$ be a non-negative integer denoting future periods. Suppose at each period $t \geq 0$, the stock-price in $t+1$ obeys the random progression:

$$
X_{t+1}= \begin{cases}X_{t} \cdot \varphi & \text { w.p. } p \\ X_{t} \cdot \varphi^{-1} & \text { w.p. } q=1-p .\end{cases}
$$

The process described above leads to a binomial conditional p.m.f. Let $t>0$ be an arbitrary future period and define the set $E_{2}^{t}=\left\{X_{0} \varphi^{-t}, X_{0} \varphi^{-(t-2)}, \ldots, X_{0} \varphi^{t-2}, X_{0} \varphi^{t}\right\}$ to be the set of possible values of the stock $t$ periods into the future. Clearly $E_{2}^{t} \subset E_{2}$. By construction, $E_{2}$ does not have any absorbing states and the conditional p.m.f of $X_{t}$ is given by:

$$
\Phi^{g}(t, j, p)=\mathbb{P}\left[X_{t}=X_{0} \cdot \varphi^{j} \mid X_{0}\right]=\left(\begin{array}{c}
t \\
\phi(j)
\end{array}\right) q^{t-\phi(j)} p^{\phi(j)}
$$

where $j \in\{-t,-(t-2), \ldots,(t-2), t\}$ and $\phi(j)=(t+j) / 2$ is the number of times the random process has moved forward in $t$ units of time.

\section{Pricing and Optimal Exercise Under the Geometric Random Walk}

Under the geometric random walk scenario, we wish to solve the problem:

$$
\begin{array}{lll}
\min \sum_{j \in \mathbb{Z}} v_{j} & & \\
\text { subject to } & v_{j} \geq f_{j}, & j \in \mathbb{Z} \\
& v_{j} \geq \alpha\left(q v_{j-1}+p v_{j+1}\right), & j \in \mathbb{Z} .
\end{array}
$$


Here, $f_{j}$ is given by $\max \left\{X_{0} \cdot \varphi^{j}-S, 0\right\}$. Constraints (1) and (2), defined over the set of integers, again correspond to the majorant and excessive properties of the value function. For the solution of P3 to coincide with the sought-after value function in Definition 2.4 we need to make sure according to Theorem 2.1 that $v$ remains finite. The expectation in the definition of the value function becomes in the present case for fixed $t$ :

$$
\begin{aligned}
\mathbb{E} & {\left[\alpha^{t}\left(X_{t}-S\right)_{+} \mid X_{0}=x\right] } \\
& =\alpha^{t} \sum_{j \in\{-t,-(t-2), \ldots,(t-2), t\}}\left(\begin{array}{c}
t \\
\phi(j)
\end{array}\right) q^{t-\phi(j)} p^{\phi(j)}\left(x \varphi^{j}-S\right)_{+} .
\end{aligned}
$$

For the value function to remain finite as $t \rightarrow \infty$, the condition

$$
\alpha \varphi p<1
$$

suffices. To see this we first observe that $\phi(j)$ runs from 0 to $t$ in the summation above, and the terms in the summation with a negative exponent on $\varphi$ vanish as $t \rightarrow \infty$ since every such term is of the form $\alpha^{t}\left(t-c_{1}\right)(1-p)^{t-c_{1}} p^{\phi(j)}\left(x \varphi^{j}-S\right)_{+}$for some constants $c_{1}$ and $c_{2}$ (dependent on the term). On the other hand the terms receiving a positive exponent on $\varphi$ all have the form $\alpha^{t}\left(t-c_{3}\right)(1-p)^{\phi(j)} p^{t-c_{4}}\left(x \varphi^{j}-S\right)_{+}$ for some constants $c_{3}$ and $c_{4}$ (dependent on the term), and each such term vanishes as $t \rightarrow \infty$ provided that condition (3) holds.

Now, we shall pursue a solution strategy based on linear programming duality. Let us consider the dual problem to the original problem (P3):

$$
\begin{aligned}
& \max \sum_{j \in \mathbb{Z}} f_{j} \cdot y_{j} \\
& \text { subject to } \quad y_{j}-\alpha p z_{j-1}+z_{j}-\alpha q z_{j+1}=1, \quad j \in \mathbb{Z} \\
& y_{j} \geq 0, \quad j \in \mathbb{Z} \\
& z_{j} \geq 0, \quad j \in \mathbb{Z}
\end{aligned}
$$

which yield the complementary-slackness (CS) conditions:

$$
\begin{aligned}
\left(f_{j}-v_{j}\right) \cdot y_{j} & =0, & & j \in \mathbb{Z} \\
\left(\alpha\left(p v_{j+1}+q v_{j-1}\right)-v_{j}\right) \cdot z_{j} & =0, & & j \in \mathbb{Z} \\
v_{j} \cdot\left(1-y_{j}-z_{j}+\alpha\left(p z_{j-1}+q z_{j+1}\right)\right) & =0, & & j \in \mathbb{Z} .
\end{aligned}
$$

We are interested in finding the optimal solution to P3 in order to characterize the states to take action. Let $v^{*}$ denote the optimal solution to P3. We construct the value function under the assumption that a critical threshold denoted by $j^{*}$ exists with the properties

$$
\begin{aligned}
& v_{j}=f_{j} \quad \forall j \geq j^{*} \\
& v_{j}>f_{j} \quad \forall j<j^{*} .
\end{aligned}
$$


Assuming the existence of $j^{*}$ we now derive a closed-form expression for the corresponding value function. Since $v_{j}=\max \left\{f_{j}, \alpha\left(p v_{j+1}+q v_{j-1}\right)\right\}$ and $v_{j} \neq f_{j}$ for $j<j^{*}$, due to our assumption, we have $v_{j}=\alpha\left(p v_{j+1}+q v_{j-1}\right)$ for $j<j^{*}$. To determine the value function, then, we need to solve the second order homogeneous difference equation:

$$
v_{j}-\alpha\left(p v_{j+1}+q v_{j-1}\right)=0, \quad j<j^{*}
$$

with the boundary conditions:

$$
\begin{aligned}
v_{j^{*}} & =f_{j^{*}} \\
v_{-\infty} & =0 .
\end{aligned}
$$

Using the general solution technique given in the Appendix, we can obtain a solution for the system (12)-(14). Since we are working with a homogeneous equation, we know that $v_{j}$ is of the form $C_{+} \xi_{+}^{j}+C_{-} \xi_{-}^{j}$ where $\xi_{-}$and $\xi_{+}$are the roots:

$$
\xi_{-}=\frac{-1-\sqrt{1-4 \alpha^{2} p q}}{-2 \alpha p}, \quad \xi_{+}=\frac{-1+\sqrt{1-4 \alpha^{2} p q}}{-2 \alpha p} .
$$

From the boundary condition (14), we get:

$$
\lim _{j \rightarrow-\infty}\left(C_{+} \xi_{+}^{j}+C_{-} \xi_{-}^{j}\right)=0
$$

Note that the roots $\xi_{-}$and $\xi_{+}$are greater than and less than 1 , respectively. Since $\xi_{-}>1$, we have $\lim _{j \rightarrow-\infty} \xi_{-}^{j}=0$ which reduces the boundary condition to:

$$
\lim _{j \rightarrow-\infty} C_{+} \xi_{+}^{j}=0
$$

Clearly, this is only possible when $C_{+}=0$ since $\xi_{+}^{j}$ grows without bound with decreasing $j$. Now, utilizing the boundary (13), we find $C_{-}$to be:

$$
\begin{aligned}
C_{+} \xi_{+}^{j^{*}}+C_{-} \xi_{-}^{j^{*}} & =f_{j^{*}} \\
C_{-} \xi_{-}^{j^{*}} & =f_{j^{*}} \\
C_{-} & =\frac{f_{j^{*}}}{\xi_{-}^{j^{*}}} .
\end{aligned}
$$

The coefficients $C_{+}$and $C_{-}$, thus, lead to the value function:

$$
v_{j}^{*}=f_{j^{*}} \cdot \xi_{-}^{\left(j-j^{*}\right)}
$$


for $j<j^{*}$. For other values of $j$ we already know that it is equal to $f_{j}$. The arguments given above, therefore, suggest that we have a candidate $v^{*}$ given by:

$$
v_{j}^{*}= \begin{cases}f_{j^{*}} \cdot \xi_{-}^{j-j^{*}} & j<j^{*} \\ f_{j} & j^{*} \leq j .\end{cases}
$$

We shall prove that $v^{*}$ indeed solves $\mathrm{P} 3$ to optimality by exhibiting suitable dual feasible $\left(y^{*}, z^{*}\right)$ that are complementary to $v^{*}$.

To make a reasonable guess of the dual variables $\left(y^{*}, z^{*}\right)$, we use a similar method. To begin with, we assume that $f_{j^{*}}>0$, which makes sense because it is unlikely to have a state where the decision is to exercise the option while the pay-off is zero. The pay-off $f_{j^{*}}$ at $j^{*}$ being positive implies, by definition of $v^{*}$, that $v_{j}^{*}>0$ for all $j \in \mathbb{Z}$. From the CS condition (7), we have $y_{j}^{*}=0$ for $j<j^{*}$. This, together with $v_{j}^{*}$ being positive implies that $z_{j}^{*}$ satisfies the non-homogeneous second order difference equation:

$$
z_{j}-\alpha\left(q z_{j+1}+p z_{j-1}\right)=1, \quad j<j^{*} .
$$

Since $v_{j}^{*} \neq \alpha\left(p v_{j+1}^{*}+q v_{j-1}^{*}\right)$ for $j \geq j^{*}$, CS condition (8) implies that $z_{j}^{*}=0$ for $j \geq j^{*}$. Furthermore, assuming that the sequence $\left\{z_{j}\right\}$ converges as $j \rightarrow-\infty$, we see from (16) that

$$
\lim _{j \rightarrow-\infty} z_{j}=\frac{1}{1-\alpha}
$$

Using these two boundary conditions, we can obtain a solution to (16). Let the particular solution be $\frac{1}{1-\alpha}$. For the homogeneous solution, we proceed as in the Appendix. Let $\zeta_{+}$and $\zeta_{-}$denote the corresponding roots derived in the Appendix. They are

$$
\zeta_{+}=\frac{-1+\sqrt{1-4 \alpha^{2} p q}}{-2 \alpha q} \quad \zeta_{-}=\frac{-1-\sqrt{1-4 \alpha^{2} p q}}{-2 \alpha q} .
$$

The two boundary conditions give the following system, which needs to be solved to determine $z_{j}^{*}$ :

$$
\begin{gathered}
C_{+} \zeta_{+}^{j^{*}}+C_{-} \zeta_{-}^{j^{*}}+\frac{1}{1-\alpha}=0 \\
\lim _{j \rightarrow-\infty}\left(C_{+} \zeta_{+}^{j}+C_{-} \zeta_{-}^{j}\right)+\frac{1}{1-\alpha}=\frac{1}{1-\alpha}
\end{gathered}
$$

Since $\zeta_{+}<1$ and $\zeta_{-}>1$, a line of reasoning similar to the case of $v^{*}$ applied to (18) reveals that $C_{+}=0$. Using this identity and (17), we find $C_{-}$to be:

$$
C_{-}=-\left(\frac{1}{1-\alpha}\right) \zeta_{-}^{-j^{*}}
$$


The dual variables $z^{*}$ associated with the candidate optimal solution $v^{*}$ are therefore given by the following simple formula:

$$
z_{j}^{*}= \begin{cases}\frac{1}{1-\alpha}\left(1-\zeta_{-}^{j-j^{*}}\right) & j<j^{*} \\ 0 & j^{*} \leq j\end{cases}
$$

The second set of dual variables, $y^{*}$, are also determined using the CS conditions. Since $v_{j} \neq f_{j}$ for $j<j^{*}$, from (7) we have $y_{j}=0$ for $j<j^{*}$. For $j>j^{*}$ we have $z_{j}=0$ which, in turn, implies that $y_{j}=1$ due to (9). Finally, for $j=j^{*}$ the same equation tells us that $1-y_{j^{*}}+\alpha p z_{j^{*-1}}=0$. The resulting piecewise function is:

$$
y_{j}^{*}= \begin{cases}0 & j<j^{*} \\ 1+\alpha p z_{j^{*}-1}^{*} & j=j^{*} \\ 1 & j>j^{*} .\end{cases}
$$

We have constructed a pair of candidate solutions for P3 and D3 assuming that a candidate threshold value $j^{*}$ exists. We now show that the existence of this critical state is guaranteed only when the parameters chosen for the model satisfy certain conditions. First let us make the following assumption:

Assumption 1 There exists a $j_{S} \in \mathbb{Z}$ such that $S=X_{0} \cdot \varphi^{j_{S}}$.

Note that the above assumption is only made for convenience in the analysis to follow. Removing the assumption leads to more complicated notation. It is also easy to see that the solution to the optimal stopping problem changes very little when the pay-off $\left(X_{0} \varphi^{j}-S\right)_{+}$has $S$ perturbed by a very small amount such that it falls in an interval $\left[X_{0} \varphi^{j_{S}}, X_{0} \varphi^{j_{S}+1}\right)$. One could replace an arbitrary strike $S$ by one of the form in the assumption. As a result, in some of our numerical examples (such as the example in Sect. 5) we do not use this assumption.

The following lemma restricts the existence of $j^{*}$ to a certain condition:

Lemma 4.1 A critical value $j^{*} \in \mathbb{Z}^{++}$defined as:

$$
j^{*}= \begin{cases}j_{S}+1 & \text { if } \frac{f_{j_{S}+2}}{f_{j_{S}+1}} \leq \xi_{-} \\ \max \left\{k: \frac{f_{k}}{f_{k-1}}>\xi_{-}\right\} & \text {otherwise }\end{cases}
$$

exists if and only if $\varphi<\xi_{-}$, where $\xi_{-}$is given by

$$
\xi_{-}=\frac{-1-\sqrt{1-4 \alpha^{2} p q}}{-2 \alpha p} .
$$

Proof To begin, let us observe that $f_{j}>0$ when $j \geq j_{S}+1$ and thus the ratio $\frac{f_{j}}{f_{j-1}}$ is only defined when $j \geq j_{S}+2$. The ratio is also monotonically decreasing. Furthermore, it converges to $\varphi$ as $j$ tends to infinity and $\frac{f_{j}}{f_{j-1}}>\varphi$ for all $j \geq j_{S}+2$. 
Now, suppose that $j^{*}$ as defined above exists. Then, we either have $j^{*}=j_{S}+1$ or $j^{*}=\max \left\{k: \frac{f_{k}}{f_{k-1}}>\xi_{-}\right\}$. If $j^{*}=j_{S}+1, \frac{f_{j_{S}+2}}{f_{j_{S}+1}}>\varphi$ and the definition of $j^{*}$ in (21) together imply:

$$
\xi_{-} \geq \frac{f_{j_{S}+2}}{f_{j_{S}+1}}>\varphi
$$

If, on the other hand, $j^{*}=\max \left\{k: \frac{f_{k}}{f_{k-1}}>\xi_{-}\right\}$, we have $\frac{f_{j^{*}+1}}{f_{j^{*}}} \leq \xi_{-}$. Suppose, for a contradiction, that $\xi_{-} \leq \varphi$. Since $\frac{f_{j}}{f_{j-1}}>\varphi$ for all $j \geq j_{S}+2$, we also have $\frac{f_{j^{*}+1}}{f_{j^{*}}}>\varphi$. This contradicts with $\frac{f_{j^{*}+1}}{f_{j^{*}}} \leq \xi_{-} \leq \varphi$. Thus, the condition $\varphi<\xi_{-}$is necessary for the existence of $j^{*}$.

In order to show that it is also sufficient, let us suppose $\varphi<\xi_{-}$. The existence of $j_{S}$ is guaranteed by our starting assumption. Therefore, when $\frac{f_{j_{S}+2}}{f_{j_{S}+1}} \leq \xi_{-}$, we can use $j^{*}=j_{S}+1$ which readily shows the existence of $j^{*}$. On the other hand, suppose that $\frac{f_{j_{S}+2}}{f_{j_{S}+1}}>\xi_{-}$. Since the sequence

$$
\left\{\frac{f_{j}}{f_{j-1}}, j \geq j_{S}+2\right\}
$$

is a monotonically decreasing sequence whose limit is $\varphi, \varphi<\xi_{-}$implies that there exist finitely many $j \in \mathbb{Z}$ with $j \geq j_{S}+2$ such that $\frac{f_{j}}{f_{j-1}}>\xi_{-}$. The existence of $j^{*}=\max \left\{k: \frac{f_{k}}{f_{k-1}}>\xi_{-}\right\}$, then, follows since the maximum of a finite set always exists. Hence, $\varphi<\xi_{-}$is also a sufficient condition for the existence of $j^{*}$, which completes the proof.

Now we need to show that $v^{*}$ defined in terms of the critical threshold $j^{*}$ is in fact the optimal solution to problem P3. To show this, we need to show (a) $v^{*}$ is primal feasible, (b) $\left(y^{*}, z^{*}\right)$ is dual feasible, and (c) $v^{*}, y^{*}$ and $z^{*}$ together satisfy the CS conditions.

Lemma 4.2 The candidate solution $v^{*}$ is feasible for $P 3$ if and only if $p$ and $\alpha$ are chosen such that

$$
\varphi^{j^{*}+1} \geq\left[\frac{1-\alpha}{1-\alpha p \varphi-\alpha q \varphi^{-1}}\right] \varphi^{j_{S}} .
$$

Proof We first show that the given condition is necessary for feasibility. Let us assume that $v^{*}$ as given in (15) is feasible to P3. Then, $v^{*}$ must satisfy constraints (2). This implies that the system of inequalities

$$
v_{j^{*}+k}^{*} \geq \alpha p v_{j^{*}+k+1}^{*}+\alpha q v_{j^{*}+k-1}^{*}, \quad k=1,2,3, \ldots
$$


hold with $v_{j}^{*}=f_{j}$. By substituting the values of $f_{j}$ into the above inequalities and rearranging the terms, one obtains the following system of inequalities:

$$
\varphi^{j^{*}+k} \geq\left[\frac{1-\alpha}{1-\alpha p \varphi-\alpha q \varphi^{-1}}\right] \varphi^{j_{S}}, \quad j=1,2,3, \ldots
$$

which also implies that the given condition holds. This shows that the given condition is necessary for the feasibility of $v^{*}$.

Next, we show that it is also sufficient for feasibility. Suppose that the condition above holds. We first concentrate on constraints (2). Let $j>j^{*}$. Since $\varphi^{j^{*}+k} \geq \varphi^{j^{*}+1}$ for all $k \geq 1$, we have:

$$
\varphi^{j^{*}+k} \geq \varphi^{j^{*}+1} \geq\left[\frac{1-\alpha}{1-\alpha p \varphi-\alpha q \varphi^{-1}}\right] \varphi^{j_{S}}, \quad j=1,2,3, \ldots
$$

which follows from the given condition. As noted in the first part of the proof, this system is equivalent to having:

$$
f_{j^{*}+k} \geq \alpha p f_{j^{*}+k+1}+\alpha q f_{j^{*}+k-1}, \quad k=1,2,3, \ldots
$$

which shows that the constraints (2) are satisfied with $v_{j}^{*}=f_{j}$ for $j>j^{*}$. When $j<j^{*}$, on the other hand, we already have $v_{j}^{*}=\alpha p v_{j+1}^{*}+\alpha q v_{j-1}^{*}$ since $v^{*}$ is necessarily the solution to this difference equation. Therefore, it remains to check for the case when $j=j^{*}$. Consider the solution of the difference equation:

$$
\begin{aligned}
\omega_{j} & =\alpha p \omega_{j+1}+\alpha q \omega_{j-1} \\
\omega_{j^{*}} & =f_{j^{*}} \\
\lim _{j \rightarrow-\infty} \omega_{j} & =0
\end{aligned}
$$

which extends over the set of integers. Then, $\omega_{j^{*}+1}=f_{j^{*}} \xi_{-}$. Since $\omega_{j^{*}}=\alpha p \omega_{j^{*}+1}+$ $\alpha q \omega_{j^{*}-1}$ and $\omega_{j^{*}-1}=v_{j^{*}-1}^{*}, f_{j^{*}}=\omega_{j^{*}} \geq \alpha p f_{j^{*}+1}+\alpha q v_{j^{*}-1}^{*}$ if and only if $f_{j^{*}+1} \leq$ $\omega_{j^{*}+1}=f_{j^{*}} \xi_{-}$. This means that it is sufficient to check $\frac{f_{j^{*}+1}}{f_{j^{*}}} \leq \xi_{-}$in order to show feasibility of $v^{*}$ for constraints (2) at $j=j^{*}$. Recall that $j^{*}$ may attain one of the two values defined in (21). First, suppose $j^{*}=j_{S}+1$. Then, by definition of $j^{*}$, we have $\frac{f_{j^{*}+1}}{f_{j^{*}}}=\frac{f_{j_{S}+2}}{f_{j_{S}+1}} \leq \xi_{-}$, hence the desired result. Now, let $j^{*}=\max \left\{k: \frac{f_{k}}{f_{k-1}}>\right.$ $\left.\xi_{-}\right\}$. Definition of $j^{*}$ in this case implies that $j^{*}$ is the maximum integer $k$ with the property $\frac{f_{k}}{f_{k-1}}>\xi_{-}$which further implies that for $j^{*}+1$, we have $\frac{f_{j^{*}+1}}{f_{j^{*}}} \leq \xi_{-}$. Thus, we can conclude (2) is satisfied with $v_{j}^{*}$ at $j=j^{*}$ which completes the feasibility of $v^{*}$ for constraints (2) for all $j \in \mathbb{Z}$.

Now, we turn our attention to the constraints (1). Note that, by definition of $v^{*}$, these are satisfied trivially for $j \geq j^{*}$. So, let $j<j^{*}$. Then, $v_{j}^{*}=f_{j^{*}} \xi_{-}^{j-j^{*}}$. Under the assumption that $j^{*}$ exists, we have $j^{*} \geq j_{S}+1$ which implies that $f_{j^{*}}>0$. Then, it follows that $v_{j}^{*}>0$. Note that for any $j \leq j_{S}$, we have $f_{j}=0$ and thus $v_{j}^{*}>f_{j}$ which 
is sufficient for the feasibility of (1). Therefore, it remains to check feasibility for the values of $j$ where $j_{S}<j<j^{*}$. In order to do this, let us consider the difference

$$
D=v_{j}^{*}-f_{j}
$$

for $j_{S}<j<j^{*}$. We have:

$$
\begin{aligned}
D & =f_{j^{*}} \xi_{-}^{j-j^{*}}-f_{j} \\
& =\left(X_{0} \varphi^{j^{*}}-S\right) \xi_{-}^{j-j^{*}}-\left(X_{0} \varphi^{j}-S\right) \\
& =X_{0}\left(\varphi^{j^{*}} \xi_{-}^{j-j^{*}}-\varphi^{j}\right)+S\left(1-\xi_{-}^{j-j^{*}}\right) .
\end{aligned}
$$

Note that we need to show $D \geq 0$. By the choice of $j$ and the fact that $\xi_{-}>1$, the second term in $D$ above is always strictly positive. If the first term is also greater than or equal to 0 , we are done. So, suppose that we have $X_{0}\left(\varphi^{j^{*}} \xi_{-}^{j-j^{*}}-\varphi^{j}\right)<0$. In this case, in order for $D \geq 0$, we need:

$$
X_{0}\left(\varphi^{j}-\varphi^{j^{*}} \xi_{-}^{j-j^{*}}\right) \leq S\left(1-\xi_{-}^{j-j^{*}}\right) .
$$

By rearranging the terms, we can obtain:

$$
\frac{X_{0} \varphi^{j^{*}}-S}{X_{0} \varphi^{j}-S} \geq \xi_{-}^{j^{*}-j} .
$$

Note that by the choice of $j^{*}$, for any $j_{S}<j<j^{*}$ we have $\left(j^{*}-j\right)$ inequalities satisfying:

$$
\begin{gathered}
\frac{f_{j+1}}{f_{j}} \geq \xi_{-} \\
\frac{f_{j+2}}{f_{j+1}} \geq \xi_{-} \\
\vdots \\
\frac{f_{j^{*}}}{f_{j^{*}-1}} \geq \xi_{-}
\end{gathered}
$$

Since all terms in these inequalities are positive, the inequality obtained by multiplying all terms in both sides of these inequalities does not change their direction. This operation will yield:

$$
\frac{f_{j^{*}}}{f_{j}} \geq \xi_{-}^{j^{*}-j}
$$

which is precisely the desired property for $D$ to be non-negative. Since $D \geq 0$, then, we have $v_{j}^{*} \geq f_{j}$ for all $j_{S}<j<j^{*}$, which concludes that constraints (1) are satisfied with $v^{*}$ for all $j \in \mathbb{Z}$. Since we have also shown that (2) are also satisfied, we will 
conclude that the condition presented in this lemma is a sufficient condition for the feasibility of $v^{*}$ to $\mathrm{P} 3$, which completes the proof.

Next, we show that the candidate dual solution $\left(y^{*}, z^{*}\right)$ is feasible in D3, which is needed to ascertain optimality of $v^{*}$.

Lemma 4.3 The pair of dual variables $y^{*}$ and $z^{*}$ given in (20) and (19) respectively is a feasible solution to D3.

Proof Let us first consider constraints (6). Note that for $j \geq j^{*}$, we have $z_{j}^{*}=0$ which satisfies non-negativity of $z^{*}$. Now, let $j<j^{*}$. Since $j<j^{*}$ and $\varphi>1$, we have $\varphi^{j-j^{*}} \in(0,1)$. Then, since $\alpha<1$, both $\frac{1}{1-\alpha}$ and $\left(1-\varphi^{j-j^{*}}\right)$ are strictly positive, which implies that $z_{j}^{*}>0$ for $j<j^{*}$. Therefore, $z_{j}^{*}$ satisfies constraints (6) for all $j \in \mathbb{Z}$.

Now, we show $y^{*}$ satisfies constraints (5). By definition of $y^{*}$, for any $j \neq j^{*}$ we already have $y^{*} \geq 0$. Thus, the only case to verify is when $j=j^{*}$. Since $z_{j}^{*} \geq 0$ for any $j \in \mathbb{Z}$, we have $z_{j^{*}-1}^{*} \geq 0$. Then, $y_{j^{*}}^{*}=1+\alpha p z_{j^{*}-1}^{*} \geq 0$ which concludes that $y_{j}^{*} \geq 0$ for all $j \in \mathbb{Z}$ and that $y^{*}$ satisfies constraints (5).

Finally, we show that $y^{*}$ and $z^{*}$ taken together satisfy constraints (4). First, let $j<j^{*}$. Then, by definition, $y_{j}^{*}=0$. The LHS of constraints (4), therefore, reduce to $z_{j}-\alpha p z_{j-1}-\alpha q z_{j+1}$. Since $z^{*}$ solves the difference equation $z_{j}-\alpha\left(q z_{j+1}+\right.$ $\left.p z_{j-1}\right)=1$, we have $z_{j}^{*}-\alpha\left(q z_{j+1}^{*}+p z_{j-1}^{*}\right)=1$ which shows that (4) are satisfied for $j<j^{*}$. Now, suppose that $j>j^{*}$. In this case, we have $y_{j}^{*}=1$ and $z_{j}^{*}=0$. Thus,

$$
y_{j}^{*}-\alpha p z_{j-1}^{*}+z_{j}^{*}-\alpha q z_{j+1}^{*}=1-0+0-0=1
$$

which shows that (4) are also satisfied when $j>j^{*}$. Then, it remains to check they also hold when $j=j^{*}$. But since $y_{j^{*}}^{*}=1+\alpha p z_{j^{*}-1}^{*}, z_{j^{*}}^{*}=0$ and $z_{j^{*}+1}^{*}=0$, we have:

$$
\begin{aligned}
y_{j^{*}}^{*}-\alpha p z_{j^{*}-1}^{*}+z_{j^{*}}^{*}-\alpha q z_{j^{*}+1}^{*} & =1+\alpha p z_{j^{*}-1}^{*}-\alpha p z_{j^{*}-1}^{*} \\
& =1 .
\end{aligned}
$$

Therefore, $y^{*}$ and $z^{*}$ taken together satisfy constraints (4) for all $j \in \mathbb{Z}$, which completes the proof.

Finally, we need our candidate solutions to satisfy the CS conditions (7)-(9) for optimality.

Lemma 4.4 $v^{*}, y^{*}$ and $z^{*}$ as given in (15), (20) and (19) respectively, satisfy the CS conditions (7)-(9).

Proof We first consider equations (7). For $j \geq j^{*}$, we have $v_{j}^{*}=f_{j}$. Then, $\left(f_{j}-v_{j}^{*}\right)$. $y_{j}^{*}=0$. When $j<j^{*}$, we have $y_{j}^{*}=0$, which again implies that $\left(f_{j}-v_{j}^{*}\right) \cdot y_{j}^{*}=0$. Thus, (7) are satisfied for all $j \in \mathbb{Z}$. 
Next, consider condition (8). For $j \geq j^{*}$, we have $z_{j}^{*}=0$ and the result follows similarly. For $j<j^{*}$, we know that $v_{j}^{*}$ solves $v_{j}-\alpha\left(p v_{j+1}+q v_{j-1}\right)=0, j<j^{*}$ with the corresponding boundary conditions. Thus, $v_{j}^{*}-\alpha\left(p v_{j+1}^{*}+q v_{j-1}^{*}\right)=0$. It follows, then, (8) are also satisfied by the choice of $v^{*}$ and $z^{*}$.

Finally, we check (9). From feasibility of $y^{*}$ and $z^{*}$, we have

$$
y_{j^{*}}^{*}-\alpha p z_{j^{*}-1}^{*}+z_{j^{*}}^{*}-\alpha q z_{j^{*}+1}^{*}=1
$$

for all $j \in \mathbb{Z}$. Then, $v_{j}^{*} \cdot\left(1-y_{j}^{*}-z_{j}^{*}+\alpha\left(p z_{j-1}^{*}+q z_{j+1}^{*}\right)\right)=0$. Therefore, (9) are also satisfied by the choice of $v^{*}, y^{*}$ and $z^{*}$; and we conclude that all CS conditions hold.

Using these properties for the candidate solution $v^{*}$, we can now show that it is indeed optimal to problem P3. The following theorem establishes this fact, however, it should be noted that it is valid whenever an appropriate $j^{*}$ exists. Recalling the result in Lemma 4.1, we know that such a $j^{*}$ exists only when $\varphi<\xi_{\text {- which also }}$ guarantees the agreement between the value function and $v^{*}$.

Theorem 4.1 Under Assumption 1 , let $\varphi<\xi_{-}$where $\xi_{-}$is given by

$$
\xi_{-}=\frac{-1-\sqrt{1-4 \alpha^{2} p q}}{-2 \alpha p} .
$$

Then, the solution $v^{*}$ given by (15) is optimal to problem P3 if and only if $p$ and $\alpha$ are chosen such that

$$
\varphi^{j^{*}+1} \geq\left[\frac{1-\alpha}{1-\alpha p \varphi-\alpha q \varphi^{-1}}\right] \varphi^{j_{S}} .
$$

Furthermore, $v^{*}$ coincides with the value function in Definition 2.4.

Proof Showing the given condition is necessary for optimality is straightforward. Suppose $v^{*}$ is optimal to P3. Then, $v^{*}$ must be feasible. By Lemma 4.2, then, we can conclude that the given condition must hold, which is the same for saying it is a necessary condition to optimality.

Now, we will show that it is also sufficient under the assumption that $j^{*}$ exists. Suppose this condition holds. This is enough, by Lemma 4.2, for feasibility to problem P3. Furthermore, we know that the pair of dual variables $\left(y^{*}, z^{*}\right)$ are also feasible to P3, by Lemma 4.3. Therefore, it remains to show that they satisfy CS conditions and there is no duality gap between the objective functions $P\left(v^{*}\right)$ and $D\left(y^{*}, z^{*}\right)$. But we have already shown, through Lemma 4.4, that these solutions satisfy the CS conditions. Hence, all requirements for optimality are satisfied as long as the given condition in the statement of the theorem holds. Therefore, the choice of $v^{*}$ solves P3 to optimality, as long as an appropriate $j^{*}$ exists.

Now, the condition $\varphi<\xi_{-}$is equivalent to the condition

$$
\alpha \varphi p<1+\frac{\alpha}{\varphi}(p-1)
$$


after some straightforward algebraic manipulation, which implies the condition (3) for finiteness of $v$. Hence, by Theorem $2.1 v^{*}$ coincides with the value function.

The above theorem is particularly useful in identifying the optimal stopping region when the underlying stock-price follows a geometric random walk on $\mathbb{R}^{++}$. With its structure, the function $v^{*}$ suggests that there exists a critical point $j^{*}$ identifying a corresponding state $X_{0} \cdot \varphi^{j^{*}} \in E_{2}$, which separates the state space into subsets. Whenever the stock price is less than this critical threshold, the value per state is strictly greater than the current pay-off, implying that the decision to exercise should be delayed. On the other hand, when the price of the stock is greater than or equal to this critical value, the value function gives the same amount with the existing pay-off, which means that it is no longer meaningful to hold the option for one more period.

A key observation in the scenario studied in this paper is that, the existence of a critical state is not guaranteed. By Lemma 4.1, we know that the critical value to identify $v^{*}$ does not always exist: the existence is guaranteed only when $\varphi<\xi_{-}$, which is a condition fully dependent on the parameters of the problem instance. It states that the upward movement in the stock price must be bounded with a quantity dependent on both the probability distribution of the random progression and the discount factor $\alpha$. The condition also guarantees that the optimal $v^{*}$ coincides with the sought-after value function.

\section{Numerical Illustrations}

The parameters for the examples are selected as follows (note that we do not satisfy Assumption 1):

$$
X_{0}=10, \quad \alpha=0.999, \quad \varphi=1.01, \quad S=12 .
$$

The pay-off for this particular call option is given in Fig. 1.

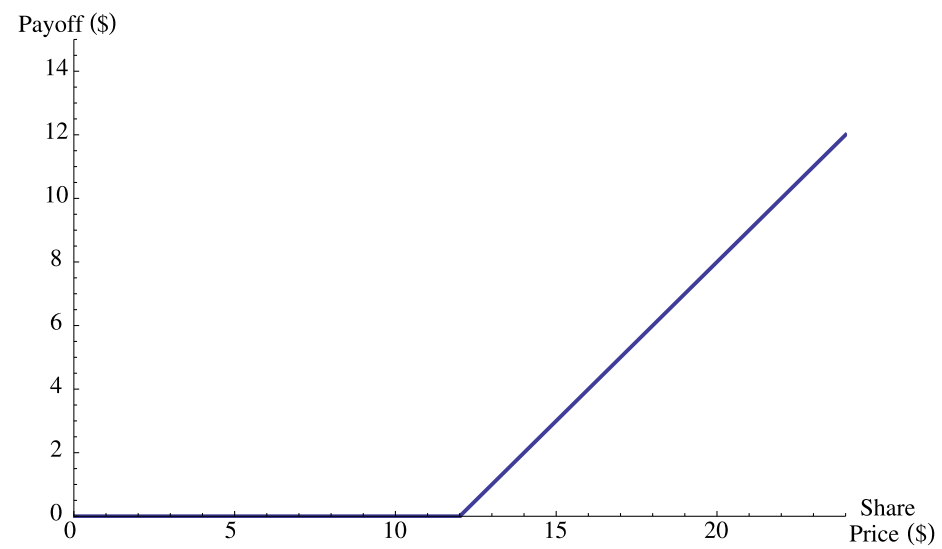

Fig. 1 Pay-off for a Call Option where $S_{0}=10, \varphi=1.01, S=12$ 
We use three different probability measures in order to show the change in the value function under varying transition probabilities. Suppose $p_{1}=0.50, p_{2}=0.52$ and $p_{3}=0.54$. Recall that as we increase $p, \xi_{-}$decreases and for $p$ close to $1, \xi_{-}$ also tends to 1 . The behaviour of $\xi_{-}$, thus, creates an upper bound for the forward probability. Since our previous analysis revealed that we must have $\varphi<\xi_{\text {- in order }}$ for a meaningful $j^{*}$ to exist (and we have set $\varphi=1.01$ for this post), we cannot test the model under larger forward probabilities. (For instance, when $p=0.60$ we have $\xi_{-}=0.00496<1.01$.)

Note that the optimal decision is to choose an integer satisfying:

$$
j^{*}= \begin{cases}j_{S}+1 & \text { if } \frac{f_{j_{S}+2}}{f_{j_{S}+1}} \leq \xi_{-} \\ \max \left\{k: \frac{f_{k}}{f_{k-1}}>\xi_{-}\right\} & \text {otherwise }\end{cases}
$$

and calculate the stock price (which is a function of $j$ ) with this critical integer as the optimal threshold of exercise. With the above set of probabilities, we have:

$$
\xi_{-}^{0.50}=1.04576, \quad \xi_{-}^{0.52}=1.02022, \quad \xi_{-}^{0.54}=1.01173 .
$$

The corresponding critical integers, in order of appearance are:

$$
j_{0.50}^{*}=44, \quad j_{0.52}^{*}=87, \quad j_{0.54}^{*}=211 .
$$

The stock values for these particular integers are approximately:

$$
X(44)=15.50, \quad X(87)=23.77, \quad X(211)=81.62 .
$$

Figure 2 shows the resulting graph for these three instances of the problem.

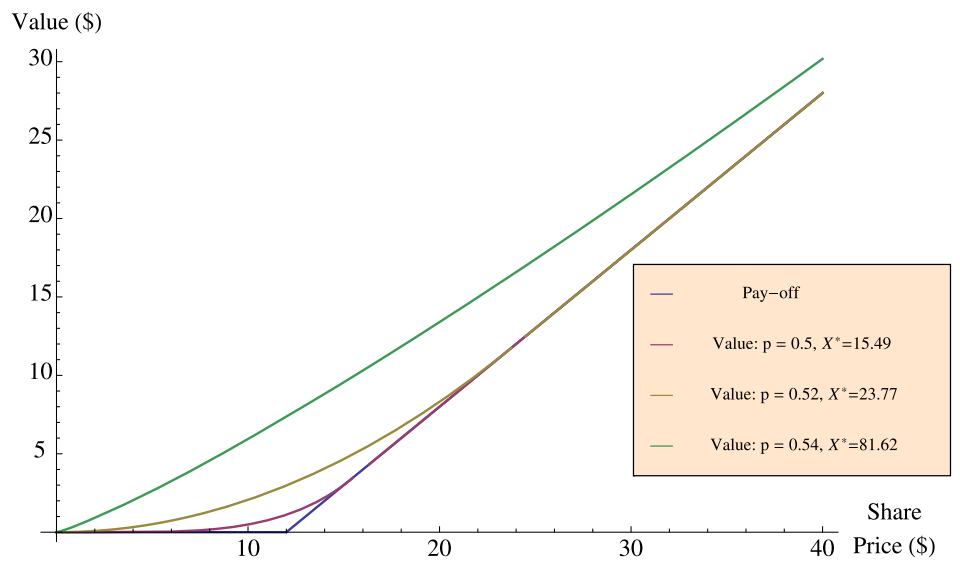

Fig. 2 Value of a American Perpetual Call under various parameters 


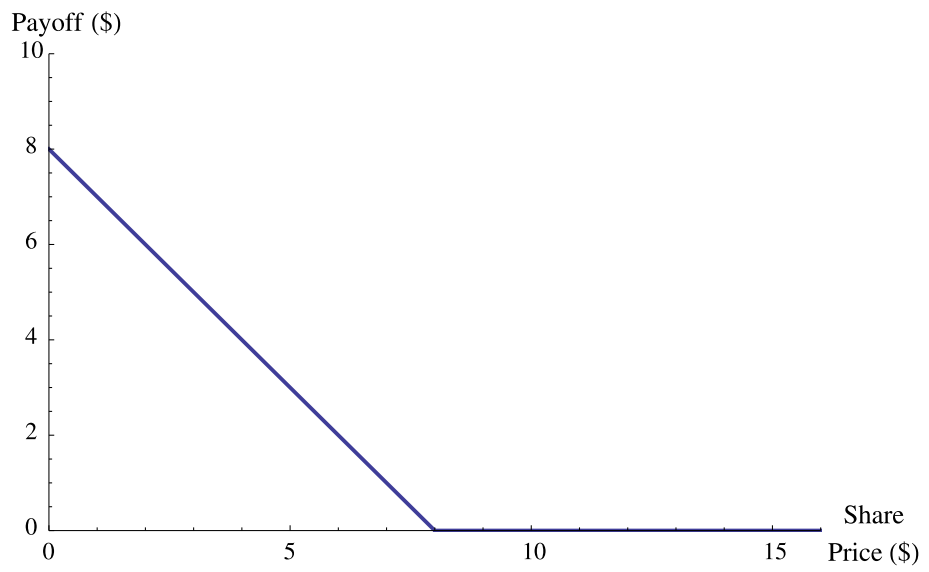

Fig. 3 The Pay-off of a Put Option for $X_{0}=10, S=8$ and $\varphi=1.01$

\section{The Case of an American Perpetual Put Option}

In this section we apply the ideas of the previous section to the American perpetual put option.

Let $S$ be the strike price for a put option and $\varphi$ be a real number slightly greater than 1. Assuming that the stock price follows the random walk $X_{t}$ on $E_{2}=\left\{X_{0} \varphi^{j}\right.$ : $j \in \mathbb{Z}$ \}, the pay-off function is:

$$
f\left(X_{t}\right)=\max \left\{S-X_{t}, 0\right\}
$$

which gives a positive value only when the stock price is less than the strike price $S$. Note also that the pay-off tends to the strike price as $j \rightarrow-\infty$ since we have:

$$
\lim _{j \rightarrow-\infty} X_{0} \varphi^{j}=0
$$

due to the choice of $\varphi$. This pay-off is shown in Fig. 3 for the values of $X_{0}=10$, $S=8$ and $\varphi=1.01$.

As in our previous approach, we will assume that the value function satisfies certain properties. Let us assume that there exists a critical point $j^{*}$ such that $\forall j \leq j^{*}$ we have $v_{j}=f_{j}$ and $\forall j>j^{*}$ we have:

$$
v_{j}=\alpha\left(p v_{j+1}+q v_{j-1}\right) .
$$

We further assume that $\lim _{j \rightarrow \infty} v_{j}=0$ which leads to the difference equation:

$$
v_{j}-\alpha\left(p v_{j+1}+q v_{j-1}\right)=0
$$

with the boundary conditions:

$$
\begin{aligned}
v_{j^{*}} & =f_{j^{*}} \\
\lim _{j \rightarrow \infty} v_{j} & =0 .
\end{aligned}
$$

The solution to this difference equation yields the desired value function. 


\subsection{Solving the Difference Equation}

Recalling the general solution of such difference equations, we can write the system:

$$
\begin{aligned}
C_{+} \xi_{+}^{j^{*}}+C_{-} \xi_{-}^{j^{*}} & =f_{j^{*}} \\
\lim _{j \rightarrow \infty}\left(C_{+} \xi_{+}^{j}+C_{-} \xi_{-}^{j}\right) & =0
\end{aligned}
$$

using the two boundary conditions above. Here, $\xi_{+}$and $\xi_{-}$are the same roots as we have used before, and given in terms of $\alpha, p$ and $q$ :

$$
\xi_{-}=\frac{-1-\sqrt{1-4 \alpha^{2} p q}}{-2 \alpha p}, \quad \xi_{+}=\frac{-1+\sqrt{1-4 \alpha^{2} p q}}{-2 \alpha p} .
$$

We know that $\xi_{-}>1$ and $\xi_{+}<1$. Thus, we have $\lim _{j \rightarrow \infty} \xi_{+}^{j}=0$. For this reason, (25) reduces to:

$$
\lim _{j \rightarrow \infty} C_{-} \xi_{-}^{j}=0
$$

which implies that $C_{-}=0$. Then, using (24) we can obtain $C_{+}=\frac{f_{j^{*}}}{\xi_{+}^{j^{*}}}$. Substituting the values of $C_{+}$and $C_{-}$and using our previous assumption, we get the value function given below:

$$
v_{j}^{*}= \begin{cases}f_{j^{*} \xi_{+}^{j-j^{*}}} & j>j^{*} \\ f_{j} & j \leq j^{*}\end{cases}
$$

This value function, dependent on the value of $j^{*}$, provides a class of functions for different values of the underlying probabilities. Now, we describe the critical exercise point $j^{*}$.

\subsection{Determining the Critical Threshold}

Note that the value function is equal to the pay-off for sufficiently small values of $j$. Thus, we seek the maximum integer where the ratio of value to pay-off is exactly 1 . Or, from the opposite direction, we seek the minimum integer $j$ such that the ratio of value to pay-off at $j+1$ is greater than 1 . In mathematical terms, we would like to find a $j^{*}$ satisfying:

$$
j^{*}=\min \left\{j: \frac{v_{j+1}}{f_{j+1}}>\frac{v_{j}}{f_{j}}\right\} .
$$

By substituting the value function into the above definition and rearranging the terms we obtain:

$$
j^{*}=\min \left\{j: \frac{f_{j+1}}{f_{j}}<\xi_{+}\right\} .
$$


Let us try to clarify this selection criterion. This formula tells us that we will compare the ratio $\frac{f_{j+1}}{f_{j}}$ to the root $\xi_{+}$and report the integer $j$ as the critical exercise point just before the ratio exceeds $\xi_{+}$. We know that $\xi_{+}$is strictly less than 1 . But we have:

$$
\lim _{j \rightarrow-\infty} \frac{f_{j+1}}{f_{j}}=\lim _{j \rightarrow-\infty} \frac{S-X_{0} \varphi^{j+1}}{S-X_{0} \varphi^{j}}=1 .
$$

Thus, there exists at most finitely many integers such that $\frac{f_{j+1}}{f_{j}}<\xi_{+}$which also implies the existence of a critical threshold value. We say at most because we do not know whether there actually are integers satisfying this relation. Note that the ratio is defined for $j<j_{S}-1$ where $j_{S}$, by assumption, is the unique integer $k$ satisfying $S=X_{0} \varphi^{k}$. Thus, the ratio, starting with $\frac{f_{j_{S}-1}}{f_{j_{S}-2}}$ could entirely remain above $\xi_{+}$. To handle this case, we update the definition of $j^{*}$ in the following way:

$$
j^{*}= \begin{cases}j_{S}-1 & \text { if } \frac{f_{j_{S}-1}}{f_{j_{S}-2}} \geq \xi_{+} \\ \min \left\{j: \frac{f_{j+1}}{f_{j}}<\xi_{+}\right\} & \text {otherwise }\end{cases}
$$

The first case in this definition is parallel to what we have derived in the case of call options under geometric random walks. It means that the trader will have to exercise as long as (s)he observes a positive pay-off.

\subsection{Dual Variables}

For the sake of completeness, we present the dual solution to our optimization problem. Following our notation, the dual solution for a put option will be:

$$
z_{j}^{*}= \begin{cases}\frac{1}{1-\alpha}\left(1-\zeta_{+}^{j-j^{*}}\right) & j<j^{*} \\ 0 & j^{*} \geq j\end{cases}
$$

and

$$
y_{j}^{*}= \begin{cases}0 & j>j^{*} \\ 1+\alpha q z_{j^{*}+1}^{*} & j=j^{*} \\ 1 & j<j^{*} .\end{cases}
$$

Note the change in the definition of $z^{*}: \zeta_{-}$has been replaced with $\zeta_{+}$. This is mainly due to the directional change in the index $j$. We now require $\lim _{j \rightarrow \infty} z_{j}^{*}=\frac{1}{1-\alpha}$, which is exactly in the opposite direction with respect to $j$, when compared to a call option properties. Also, $y^{*}$ needs to be adjusted at $j=j^{*}$ since the neighbour of $j^{*}$ which makes $z^{*}$ equal to zero is now $\left(j^{*}-1\right)$ as opposed to $\left(j^{*}+1\right)$ in the case of a call.

\subsection{An Example}

Let us use the parameters of the previous plot to calculate an optimal exercise point. To ensure existence of $j_{S}$, let $S=8.034$. Then, we have $j_{S}=-22$. For any $j<$ 
Table 1 Value of $\xi_{+}$for varying $\alpha$ and $p$

\begin{tabular}{|c|c|c|c|c|c|c|c|c|c|}
\hline \multicolumn{2}{|c|}{$\alpha=0.999$} & \multicolumn{2}{|c|}{$\alpha=0.995$} & \multicolumn{2}{|c|}{$\alpha=0.9$} & \multicolumn{2}{|c|}{$\alpha=0.75$} & \multicolumn{2}{|c|}{$\alpha=0.5$} \\
\hline$p$ & $\xi_{+}$ & $p$ & $\xi_{+}$ & $p$ & $\xi_{+}$ & $p$ & $\xi_{+}$ & $p$ & $\xi_{+}$ \\
\hline 0.1 & 0.9988 & 0.1 & 0.9938 & 0.1 & 0.8796 & 0.1 & 0.7131 & 0.1 & 0.4606 \\
\hline 0.2 & 0.9983 & 0.2 & 0.9917 & 0.2 & 0.8501 & 0.2 & 0.6667 & 0.2 & 0.4174 \\
\hline 0.3 & 0.9975 & 0.3 & 0.9877 & 0.3 & 0.8049 & 0.3 & 0.6082 & 0.3 & 0.3706 \\
\hline 0.4 & 0.9951 & 0.4 & 0.9766 & 0.4 & 0.7339 & 0.4 & 0.5363 & 0.4 & 0.3206 \\
\hline 0.5 & 0.9562 & 0.5 & 0.9046 & 0.5 & 0.6268 & 0.5 & 0.4514 & 0.5 & 0.2679 \\
\hline 0.6 & 0.6634 & 0.6 & 0.6510 & 0.6 & 0.4893 & 0.6 & 0.3575 & 0.6 & 0.2137 \\
\hline 0.7 & 0.4275 & 0.7 & 0.4233 & 0.7 & 0.3450 & 0.7 & 0.2607 & 0.7 & 0.1588 \\
\hline 0.8 & 0.2496 & 0.8 & 0.2479 & 0.8 & 0.2125 & 0.8 & 0.1667 & 0.8 & 0.1044 \\
\hline 0.9 & 0.1110 & 0.9 & 0.1104 & 0.9 & 0.0977 & 0.9 & 0.0792 & 0.9 & 0.0512 \\
\hline
\end{tabular}

-22 , we have $f_{j}>0$. Now, consider $\frac{f_{-23}}{f_{-24}}=0.5025$. According to (27), for $\xi_{+} \leq$ 0.5025 , the trader has to exercise as soon as the stock price hits $X_{0} \varphi^{-23}=7.9544$. But the choice of $\xi_{+}$depends on the underlying probability measure and the discount factor $\alpha$. Table 1 shows a selection of $\xi_{+}$values under varying $p$ and $\alpha$.

Note that as the forward probability $p$ increases, the value of $\xi_{+}$falls below the 0.5025 which implies that the trader has to exercise as soon as (s)he receives a positive value. The result is also intuitive because for a higher forward probability, the tendency to move forward reduces the expectation of a positive pay-off and pushes the critical state to exercise as low as possible.

For lower values of $p$, the exercise region should be calculated as given in the second case of (27). Since the pay-off ratio converges to 1, the existence of such a point is guaranteed.

\subsection{On the Existence of a Critical State}

The valuation of a put option under geometric random walk differs from the valuation of a call option since the pay-off for a put option is bounded whereas for a call option, we have an unbounded pay-off function. Recall that in the case of a call option, the pay-off ratio converged to $\varphi$, a parameter of the model. Thus, for sufficiently large $\varphi$, we had a convergence issue and showed that a critical point may not exist.

In the case of a put option, the pay-off ratio converges to 1, which follows from the fact that $f$ is bounded. This is almost similar to the simple random walk model, ensuring that the pay-off ratio intersects $\xi_{+}$. Thus, one does not need to worry about existence of an optimal solution.

Various exercise points with different parameters are shown in Fig. 4.

\section{Conclusions}

In this paper we analyzed the optimal exercise problem for American perpetual options, and derived a closed form valuation formula under some mild assumptions on 


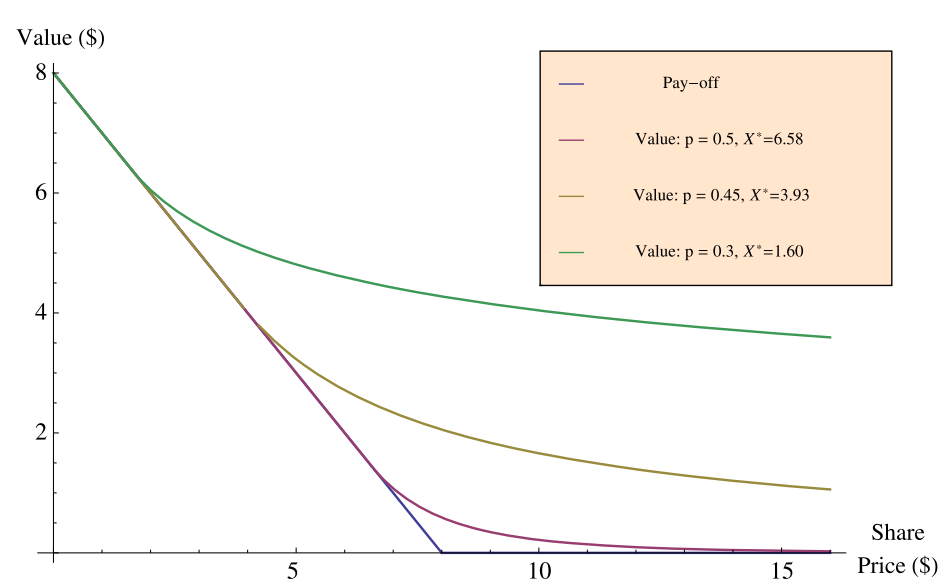

Fig. 4 Value of a Put Option under various parameters

the problem parameters. A similar analysis is applicable to American perpetual put options. Due to the particular form of the pay-off function, an optimal finite exercise policy always exists in the case of the put whereas for the call case exercise depends on the values of certain parameters.

\section{Appendix: Solution of Second Order Difference Equations with Given Boundary Conditions}

The system of linear equations,

$$
a_{0} v_{j}+a_{1} v_{j-1}+a_{2} v_{j-2}=b_{0}, \quad j \in \mathcal{I}
$$

is known as a second order linear difference equation on the index set $\mathcal{I}$ where it is convenient to consider $\mathcal{I}$ to be a subset of the set of integers. It is said to be homogeneous if $b_{0}=0$ and non-homogeneous if otherwise. We shall give, here, the solution method adopted in this work to obtain a closed form formula for the unknown $v$.

It is assumed, a priori, that the solution is of the form $\xi^{j}$. If the difference equation encountered is a homogeneous equation, we have:

$$
a_{0} \xi^{j}+a_{1} \xi^{(j-1)}+a_{2} \xi^{(j-2)}=0
$$

Dividing both sides of the equation by $\xi^{(j-2)}$, we get:

$$
a_{0} \xi^{2}+a_{1} \xi+a_{2}=0
$$

Which will have the obvious roots:

$$
\xi_{+}=\frac{-a_{1}+\sqrt{a_{1}^{2}-4 a_{0} a_{2}}}{2 a_{0}}, \quad \xi_{-}=\frac{-a_{1}-\sqrt{a_{1}^{2}-4 a_{0} a_{2}}}{2 a_{0}}
$$


The closed form formula for $v$, then, will be in the form:

$$
v_{j}=A \cdot \xi_{+}^{j}+B \cdot \xi_{-}^{j}
$$

where the coefficients $A$ and $B$ can be obtained by solving the system:

$$
\begin{aligned}
& A \cdot \xi_{+}^{j_{1}}+B \cdot \xi_{-}^{j_{1}}=v_{j_{1}} \\
& A \cdot \xi_{+}^{j_{2}}+B \cdot \xi_{-}^{j_{2}}=v_{j_{2}}
\end{aligned}
$$

with two known values of $v$. If, we have a non-homogeneous equation, we can write:

$$
v_{j}=v_{j}^{h}+\bar{v}_{j}
$$

where $v_{j}^{h}$ is the solution to the homogeneous case and $\bar{v}_{j}$ is any particular solution to the non-homogeneous case.

The technique given here can be used to solve difference equations with arbitrary boundary values. Assume we are given a difference equation of the form 30 and any two points $j_{1}, j_{2} \in \mathcal{I}$ such that $v_{j_{1}}=f_{1}$ and $v_{j_{2}}=f_{2}$. Roots of the homogeneous case can clearly be obtained with ease. Let's say these are $\lambda_{+}$and $\lambda_{-}$. The boundaries of the difference equation yield the following system:

$$
\begin{aligned}
& C_{+} \lambda_{+}^{j_{1}}+C_{-} \lambda_{-}^{j_{1}}=f_{j_{1}} \\
& C_{+} \lambda_{+}^{j_{2}}+C_{-} \lambda_{-}^{j_{2}}=f_{j_{2}}
\end{aligned}
$$

which has the solution:

$$
C_{+}=\frac{\lambda_{-}^{j_{1}} f_{j_{2}}-\lambda_{-}^{j_{2}} f_{j_{1}}}{\lambda_{-}^{j_{1}} \lambda_{+}^{j_{2}}-\lambda_{+}^{j_{1}} \lambda_{-}^{j_{2}}}, \quad C_{-}=\frac{\lambda_{+}^{j_{2}} f_{j_{1}}-\lambda_{+}^{j_{1}} f_{j_{2}}}{\lambda_{-}^{j_{1}} \lambda_{+}^{j_{2}}-\lambda_{+}^{j_{1}} \lambda_{-}^{j_{2}}}
$$

In the general sense, then, the homogeneous case has the solution

$$
v_{j}=\left(\frac{\lambda_{-}^{j_{1}} f_{j_{2}}-\lambda_{-}^{j_{2}} f_{j_{1}}}{\lambda_{-}^{j_{1}} \lambda_{+}^{j_{2}}-\lambda_{+}^{j_{1}} \lambda_{-}^{j_{2}}}\right) \lambda_{+}^{j}+\left(\frac{\lambda_{+}^{j_{2}} f_{j_{1}}-\lambda_{+}^{j_{1}} f_{j_{2}}}{\lambda_{-}^{j_{1}} \lambda_{+}^{j_{2}}-\lambda_{+}^{j_{1}} \lambda_{-}^{j_{2}}}\right) \lambda_{-}^{j}, \quad j_{1}<j<j_{2}
$$

The reader can easily verify that this general solution reduces to the solution we have just obtained for the $0-j^{*}$ instance.

In the present paper we deal with a boundary value of the form:

$$
v_{ \pm \infty}=0
$$

implying that the value function reduces asymptotically to 0 as the index value is increased without bound, e.g., the geometric random walk case where the stock price never reduces to zero, but the value function must tend to zero accompanying the price of the stock. Suppose that this leads to the boundary condition:

$$
\lim _{j \rightarrow-\infty}\left(C_{+} \lambda_{+}^{j}+C_{-} \lambda_{-}^{j}\right)=0 .
$$


If it is known that one of the roots is strictly greater than 1 , as it is the case for the model studied in the present paper, the boundary condition reduces to an equation which is in terms of the second root. Say $\lambda_{-}>1$. Clearly we have $\lim _{j \rightarrow-\infty} \lambda_{-}^{j}=0$. Then, the reduced equation will be:

$$
\lim _{j \rightarrow-\infty} C_{+} \lambda_{+}^{j}=0
$$

Note that for $\lambda_{+}<1, \lambda_{+}^{j}$ grows without bound as $j \rightarrow-\infty$. The only possibility for $C_{+}$to satisfy the boundary condition is, thus, to have the value 0 . The coefficient $C_{-}$ can, then, be solved with the knowledge of another boundary condition. Say, for some integer $j^{\prime}$, the value of $v$ is known, that is $v_{j^{\prime}}=K$ where $K$ represents this known value. $C_{-}$will clearly be equal to $K \cdot \lambda_{-}^{-j^{\prime}}$, and the solution to the homogeneous case will be:

$$
v_{j}=K \cdot \lambda_{-}^{j-j^{\prime}}
$$

The treatment of the case where $j$ tends to $\infty$ is similar. Note that the arguments presented here are valid provided that a finite $j^{\prime}$ for which the value of $v$ is known exists.

\section{References}

1. Çınlar, E.: Introduction to Stochastic Processes. Prentice Hall, New York (1975)

2. Darling, D., Liggett, T., Taylor, H.: Optimal stopping for partial sums. Ann. Math. Stat. 43, 1363-1368 (1972)

3. Deligiannidis, G., Le, H., Utev, S.: Optimal stopping for processes with independent increments, and applications. J. Appl. Probab. 46, 1130-1145 (2009)

4. Diermann, F.: Optimal stopping for processes under ambiguity via measure transformation. Master's thesis, Bielefeld University, Germany. http://erasmus-mundus.univ-paris1.fr/fichiers_etudiants/ 3160_dissertation.pdf (2010)

5. Dynkin, E.B., Yushkevich, A.A.: Markov Processes: Theorems and Problems. Plenum, New York (1969). Translated from Russian by James S. Wood

6. Helmes, K., Stockbridge, R.: Construction of the value function and stopping rules in optimal stopping of one-dimensional diffusions. Adv. Appl. Probab. 42, 158-182 (2010)

7. Hobson, D.: A survey of mathematical finance. Proc. R. Soc. A, Math. Phys. Eng. Sci. 460(2052), 3369-3401 (2004)

8. Karatzas, I.: On the pricing of american options. Appl. Math. Optim. 17, 37-60 (1988)

9. Myneni, R.: The pricing of the American option. Ann. Appl. Probab. 2(1), 1-23 (1992)

10. Samuelson, P.A.: Rational theory of warrant pricing. Ind. Manage. Rev. 6, 13-31 (1965). Appendix by H.P. McKean, pp. 32-39

11. Shreve, S.E.: Stochastic Calculus fr Finance II Continuous-Time Models. Springer, New York (2004)

12. Sødal, S.: The stochastic rotation problem: a comment. J. Econ. Dyn. Control 26, 509-515 (2002)

13. Sødal, S.: Entry and exit decisions based on a discount factor approach. J. Econ. Dyn. Control 30, 1963-1968 (2006)

14. Taksar, M.: Infinite dimensional linear programming approach to singular stochastic control. SIAM J. Control Optim. 35(2), 604-625 (1997)

15. van Moerbeke, P.: On optimal stopping and free boundary problems. Arch. Ration. Mech. Anal. 60, 101-148 (1976) 
16. Vanderbei, R., Pınar, M.Ç.: Pricing american perpetual warrants by linear programming. SIAM Rev. 51(4), 767-782 (2009)

17. Willassen, Y.: The stochastic rotation problem: A generalization of Faustmann's formula to stochastic growth. J. Econ. Dyn. Control 22, 573-596 (1998)

18. Wong, D.: Generalized Optimal Stopping Problems and Financial Markets. Addison-Wesley, Longman (1996) 